\title{
Qualitative study on the recovery journey of peers affected by severe mental illness: the experience from the "Entrelaços" program, Brazil.
}

\author{
Leonardo Palmeira, Alexandre Keusen, Elias Carim Neto, Silvana Barreto, Olga Leão, Maria T. Cavalcanti. \\ Insitute of Psychiatry, Federal University of Rio de Janeiro \\ drpalmeira@gmail.com
}

\section{Introduction}

The "Entrelaços" Program is a peer support program of the Institute of Psychiatry of the Federal University of Rio de Janeiro (IPUB), which combines psychoeducation with problem-solving therapy for patients with severe mental disorder and their families. Running since 2011, the program has enabled the formation of seven peer groups in the city of Rio de Janeiro, conducted by themselves and outside institutional spaces, creating a community support network independent of psychiatric services. Peer support has been considered strategic for psychiatric rehabilitation by embodying the paradigm shift in mental health recovery, sharing stories that serve as a model and inspiration for other patients, and exchanging information, skills and solutions that can transform lives beyond the reach of the services. ${ }^{1,2}$ The present study aims to understand the trajectory of patients who underwent the "Entrelaços" Program, from getting sick to the present moment, and what influence the participation in the program and peer groups had in this process.

\section{Methods}

Nine patients were selected for a qualitative interview that addressed 6 different aspects of their trajectories: illness, treatment, "Entrelaços" Program, recovery process, peer support group, changes in mental health services.

The interviews were audio recorded and transcribed. Content analysis was utilized by means of the ATLAS.ti software.

\section{Results}

\begin{tabular}{|c|}
\hline Illness and Treatment \\
\hline $\begin{array}{l}\text { - } \quad \text { Passivity and impotence } \\
\text { - } \quad \text { Family's unpreparedness } \\
\text { - } \text { consultation/treatment } \\
\text { - } \text { Diagnosis and stigma } \\
\text { Hazards of medication and non- } \\
\text { - Ldherence } \\
\text { Lucidity and shame/regret after } \\
\text { relapse }\end{array}$ \\
\hline
\end{tabular}

\section{"Entrelaços" and Peer Groups}

- "Teaching Physician"

- Recovery paradigm, vulnerability model, motivation and hope

- Improved communication and expressed emotion in the family

- Exchange of experiences and solutions

- Relativize problems

- Peers as models

- Helper-therapy principle

- Expansion of the social network

- Stigma reduction

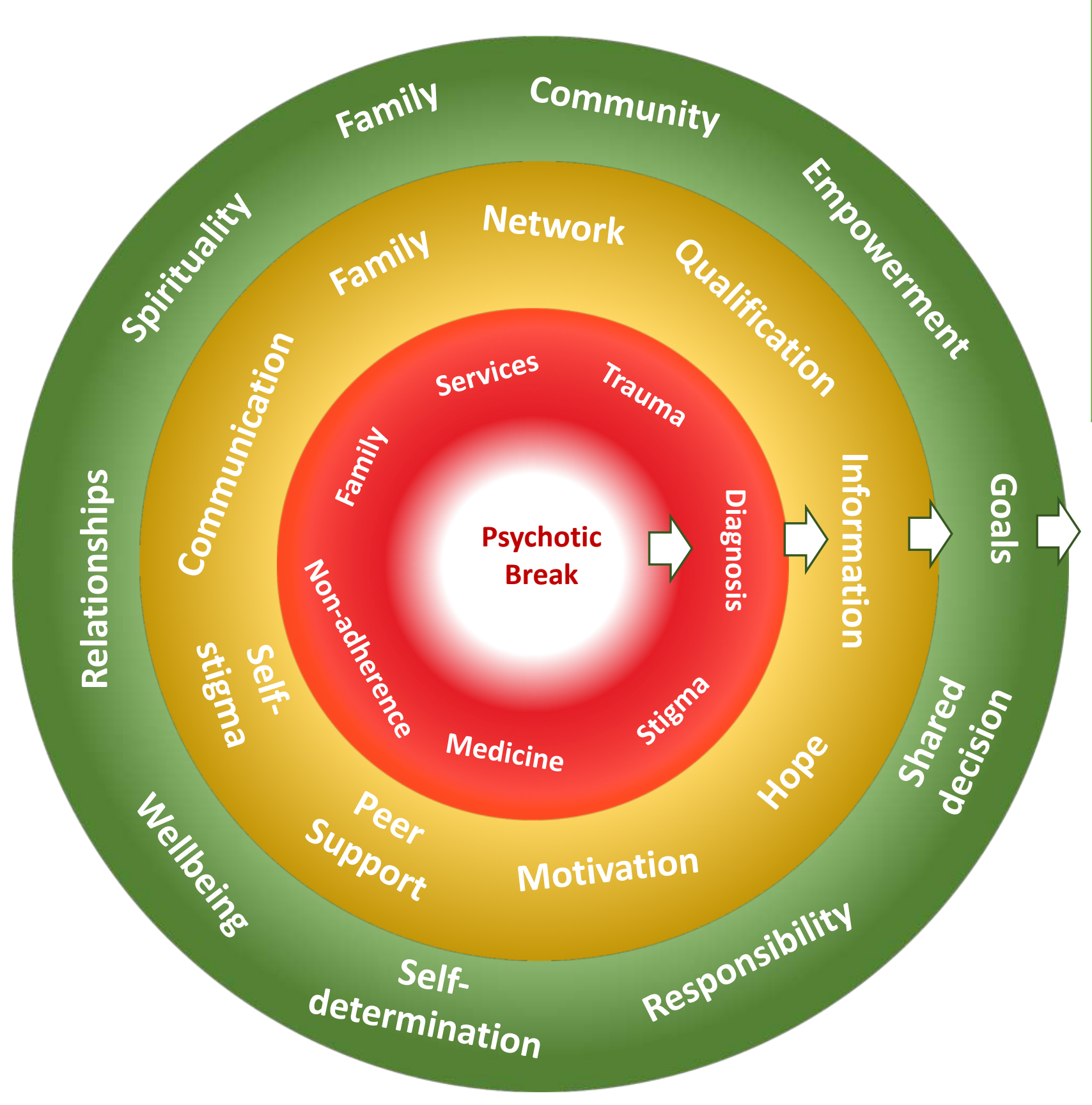

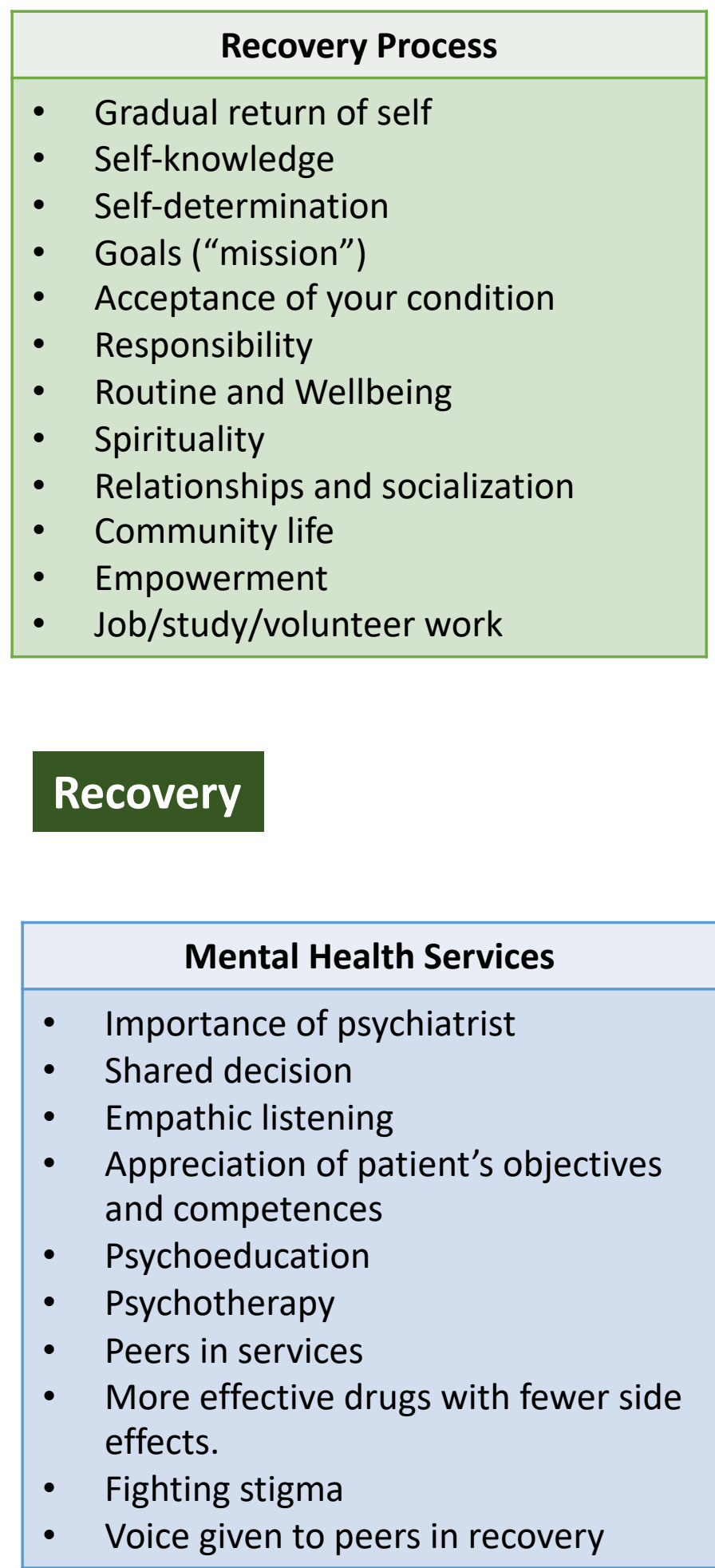

\section{Conclusions}

The illness and the beginning of the treatment were, in the experience of those patients, a cycle that was self-perpetuating due to the lack of information and guidance to the family, impersonal approach to treatment, with drugs that were harmful and often imposed on them, lack of diagnosis' understanding and stigma, which together led to non-adherence and relapses.

Participation in the program brought the information and presented them with a new vision of the disorder, the future and the possibilities for recovery, giving back to them the motivation and the hope they had lost. The development of a new expertise to deal with the problem was made possible through the exchange of experiences, problem-solving and communication skills, and the expansion of the social network where one peer supports the other. The recovery process was gradually built up throughout the program and the work with the peer group in the community, giving back to them a sense of self, allowing them to organize themselves around goals, set and take responsibility for themselves and pass to guide their lives according to their purposes. 